\section{A Pit Bull Type (PBT) aid to identification protocol clinical abstract}

\section{Adele Wharton, David Bailey, Sean Taylor, Claire Gwinnett}

University of Staffordshire, Staffordshire, UK

The determination of a Pit Bull Type breed (PBT) has proven problematic since the introduction of the Dangerous Dogs Act in the UK in 1991

A PBT is not a named breed of animal but a type of animal and the burden of proof in these cases is reversed. Any breed of dog can be seized and deemed a PBT and the burden is on the owner to prove that it isn't.

Currently the identification of Pit Bull Type (PBT) dogs relies upon a list of subjective physical characteristics taken from the American Dog Breeders Association Standard of Conformation.

The law does not require a deemed PBT to fit the description perfectly, rather it requires for a 'substantial number' of characteristics to be present so that it can be considered 'more likely' to be a PBT than any other type of dog.

Using microscopic analysis of samples of over 800 hairs and 16,000 individual measurements of known PBT hair from the USA, UK and Republic of Ireland we have compiled a database (the first of its kind) which shows that objective, noninvasive measurements of hair features can be taken in order

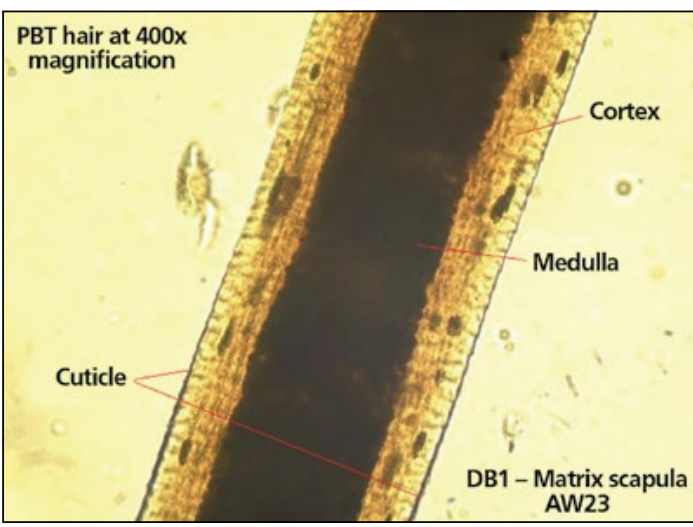

Figure 1.

to aid in the identification of a PBT dog.

In the field of human forensics, hair samples can be used to identify the ethnicity of the donor, and we have found similar physical attributes which can be used to differentiate between PBT and non-PBT hair.

These characteristics relating to the thickness of the cuticle (see Figure.1), and the relationship between the width of the cuticle, cortex and medulla can provide us with objective, repeatable and reliable characteristics that can be used alongside the existing accepted method of identification, in order to make the identification process more reliable, and to reduce the subjectivity associated with the current process.

\section{Abnormalities detected during routine examination at annual vaccination in dogs}

\section{David Williams, Agata Grudzien}

Department of Veterinary Medicine, University of Cambridge, Cambridge, UK

It is accepted by many that vaccination for canine viral infections can be given less frequently than annually and that longer intervals between vaccinations still give immunological competence. Yet yearly vaccination is recommended by most as it promotes regular clinical examination. How often though, are conditions noted at yearly vaccination examinations which would otherwise have been missed? Here we evaluated clinical notes made at vaccination consultations in six veterinary practices over one month.

Clinical records of 1080 vaccination consultations were evaluated. 391 dogs examined were neutered males, 192 entire males, 127 were entire females and 354 were neutered females while 16 were of undefined gender. 614 (57\%) animals had no observed abnormalities, 430 (40\%) had a condition observed at that examination and noted on the clinical record while for the remaining 36 (3\%) did not have recorded notes of the results of clinical examination. Of those with a condition noted at the examination, the diseases diagnosed are shown in Table 1 in order of prevalence.

This study shows that a substantial number of examinations $(40 \%)$ resulted in detection of a condition which might otherwise have gone un-recognised. It shows the value of an annual examination but does not answer the question of whether vaccination is a necessary 'draw' to persuade owners to bring their dog for its annual examination.

\section{Disease}

Number

Dental disease

Skin disease

Weight issues

Neoplastic disease

Ear disease

Orthopaedic disease

Cardiac disease

Ophthalmic disease

Anal gland disease

Urinary disease

Upper respiratory tract disease

Behaviour issues

Other

\begin{tabular}{|l|l|}
\hline 87 & 20.2 \\
\hline 66 & 15.4 \\
\hline 61 & 14.2 \\
\hline 42 & 9.8 \\
\hline 33 & 7.7 \\
\hline 31 & 7.2 \\
\hline 24 & 5.6 \\
\hline 22 & 5.1 \\
\hline 17 & 3.9 \\
\hline 8 & 1.9 \\
\hline 6 & 1.4 \\
\hline 3 & 0.7 \\
\hline 30 & 6.9 \\
\hline
\end{tabular}

Table 1. 